\title{
Pulmonary inflammatory myofibroblastic tumor with TPM4-ALK translocation
}

\author{
Katsuhiro Okuda, Takuya Watanabe, Risa Oda, Tadashi Sakane, Osamu Kawano, Hiroshi Haneda, Satoru \\ Moriyama, Ryoichi Nakanishi
}

Department of Oncology, Immunology and Surgery, Nagoya City University Graduate School of Medical Sciences, Nagoya, Japan

Correspondence to: Katsuhiro Okuda, MD, PhD. Department of Oncology, Immunology and Surgery, Nagoya City University Graduate School of Medical Science, 1 Kawasumi, Mizuho-cho, Mizuho-ku, Nagoya 467-8601, Japan. Email: kokuda@med.nagoya-cu.ac.jp.

\begin{abstract}
A 35-year-old woman with shortness of breath and cough was referred to our hospital and agreed to receive therapy for lung tumor in our hospital. Based on the findings from a bronchoscopic biopsy, she was suspected of having pulmonary inflammatory myofibroblastic tumor (IMT), but a correct diagnosis was not indicated. Right upper wedge lobectomy was performed. The findings of a pathological examination of the permanent surgical resected tissue, the ultimate diagnosis was pulmonary IMT. The immunohistochemistry of ALK using the intercalated antibody-enhanced polymer (iAEP) method was positive. We extracted the RNA from frozen surgical resected tumor tissue and proved the tropomyosin alpha-4 chain (TPM4)-ALK by 5 ' rapid amplification of cDNA end (5' RACE) and reverse transcription polymerase chain reaction. The preoperative bronchial biopsy specimen was also found to be positive for anti-ALK immunohistochemistry with the iAEP method. A molecular therapeutic drug may be useful as personalized therapy for tumors with ALK translocation as oncogenic drivers. We should examine the ALK protein expression and translocation in cases of lung cancer and IMT using an adequate ALK immunohistochemistry system. We experienced a case of pulmonary IMT with TPM4-ALK translocation.
\end{abstract}

Keywords: Pulmonary inflammatory myofibroblastic tumor (pulmonary IMT); TPM4-ALK; translocation

Submitted Jun 16, 2017. Accepted for publication Oct 10, 2017.

doi: $10.21037 /$ jtd.2017.10.103

View this article at: http://dx.doi.org/10.21037/jtd.2017.10.103

\section{Introduction}

ALK is a receptor tyrosine kinase that was discovered in anaplastic large cell lymphoma in the form of a fusion protein. Identifying patients with ALK-rearrangements is important for selecting ALK inhibitor therapy for nonsmall cell lung carcinoma (NSCLC) patients especially for advanced adenocarcinoma cases (1). We experienced a relatively rare pulmonary inflammatory myofibroblastic tumor (IMT) that needed to be distinguished from lung cancer on computed tomography (CT) imaging diagnosis. Using sensitive intercalated antibody-enhanced polymer (iAEP) immunohistochemistry, we confirmed this case as ALK positive pulmonary IMT. To confirm that the tumor has ALK translocation, the identification of the ALK partner is one of the reliable verifications. 5' rapid amplification of cDNA end (5' RACE), which is useful for identifying ALK fusion partner, identified the partner of ALK fusion to be tropomyosin alpha-4 chain (TPM4) in this case.

\section{Case presentation}

A 35-year-old never-smoker woman with shortness of breath and cough was found to have a pulmonary nodule at the right upper lung field by chest X-ray (Figure 1A) and CT (Figure 1B). She was referred and admitted to our hospital. Bronchoscopy revealed a tumor protruding from the upper lobe bronchus (Figure 1C) and bronchial biopsy specimens of the tumor showed marked inflammation and granulation with some fragments of non-neoplastic epithelium, and a diagnosis was not confirmed, only 

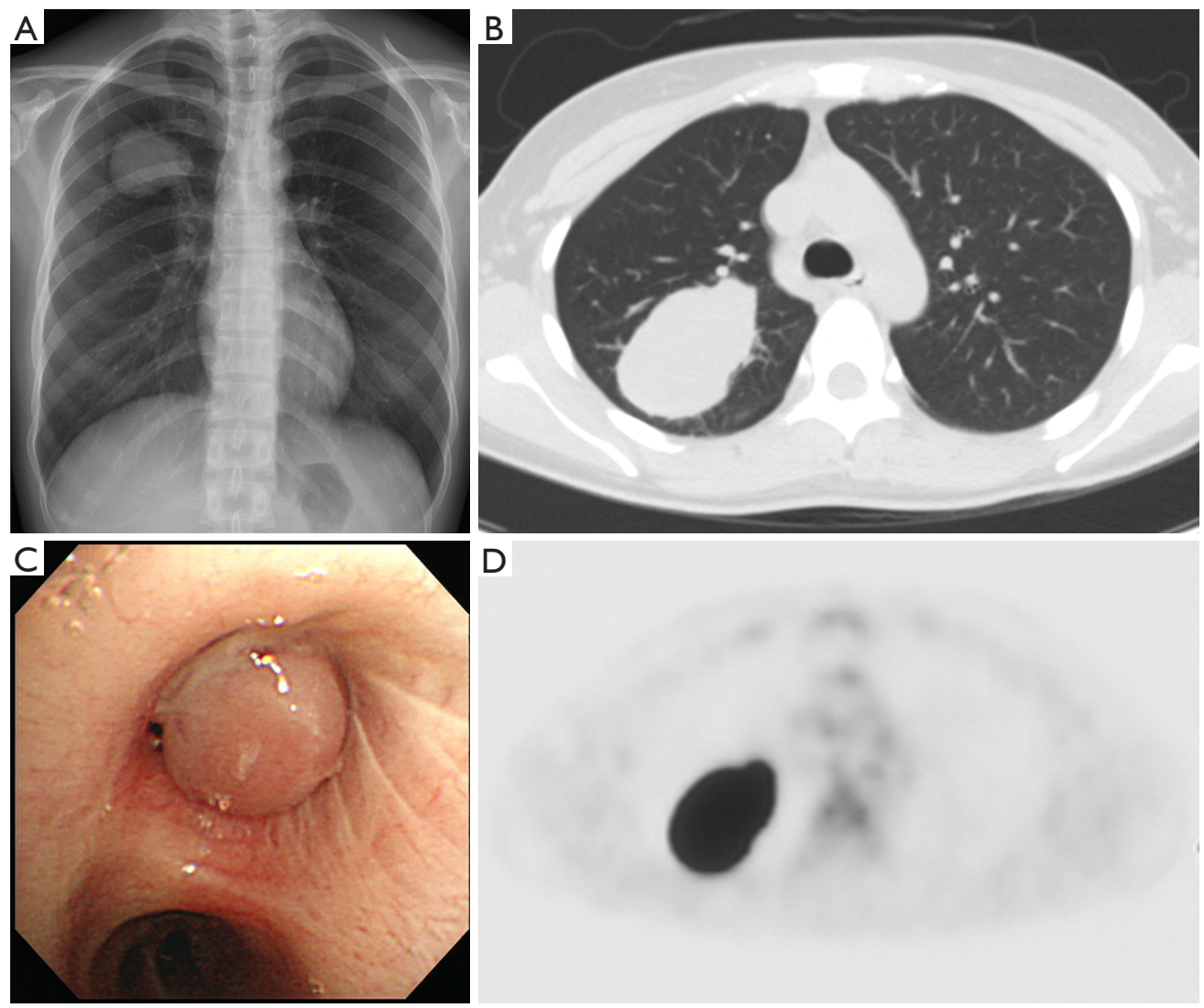

Figure 1 The findings of chest X-rays (A), chest computed tomography (B), bronchoscope (C) and positron emission tomography (D) before surgery.

suspected of being pulmonary IMT. Positron emission tomography showed a maximum standardized uptake value of 25.36 and average standardized uptake value of 17.34 in the tumor lesion (Figure 1D). Right upper wedge lobectomy and mediastinum lymph-node resection was performed. The cutting margin of bronchus was negative on a pathological examination during the operation. The tumor was macroscopically white-yellowish, and the boundary was clearly covered with bronchial epithelium (Figure 2A). According to an immunohistochemical examination of the permanent surgical resected tissue, the final diagnosis was IMT (Figure 2B). An additional immunohistochemical examination revealed ALK positivity for both the permanent surgical resected tissue and the bronchial biopsy specimen. We also detected the ALK translocation partner using RNA extracted from frozen surgically resected tumor tissue. She remains alive with neither recurrence nor distant metastasis at five years after the operation.

Tissue sections $4 \mu \mathrm{m}$ thick from the formalin-fixed and paraffin embedded surgical resected and bronchial biopsy specimens were used. Immunohistochemistry was performed using primary antibodies against ALK (5A4) and the iAEP method (2). An immunohistochemical analysis of ALK using iAEP method was positive for the surgical resected specimen (Figure 2C) and bronchoscopic biopsy specimen (Figure 2D).

5' RACE was performed using the 5' RACE System for Rapid Amplification of cDNA Ends, Version 2.0 (Invitrogen, Carlsbad, CA, USA) in accordance with the manufacturer's instructions. The primers were created using Primer 3. We extracted the RNA from surgical resected frozen tumor tissue and identified the TPM4-ALK as fusion gene by $5^{\prime}$ RACE (Figure 3A) and RT-PCR using the TPM4 exon primer (Figure $3 B$ ). A sequence analysis demonstrated that the RACE product was an in-frame fusion of the $A L K$ and TPM4 genes.

\section{Discussion}

Pulmonary IMT is a relatively rare mesenchymal tumor 

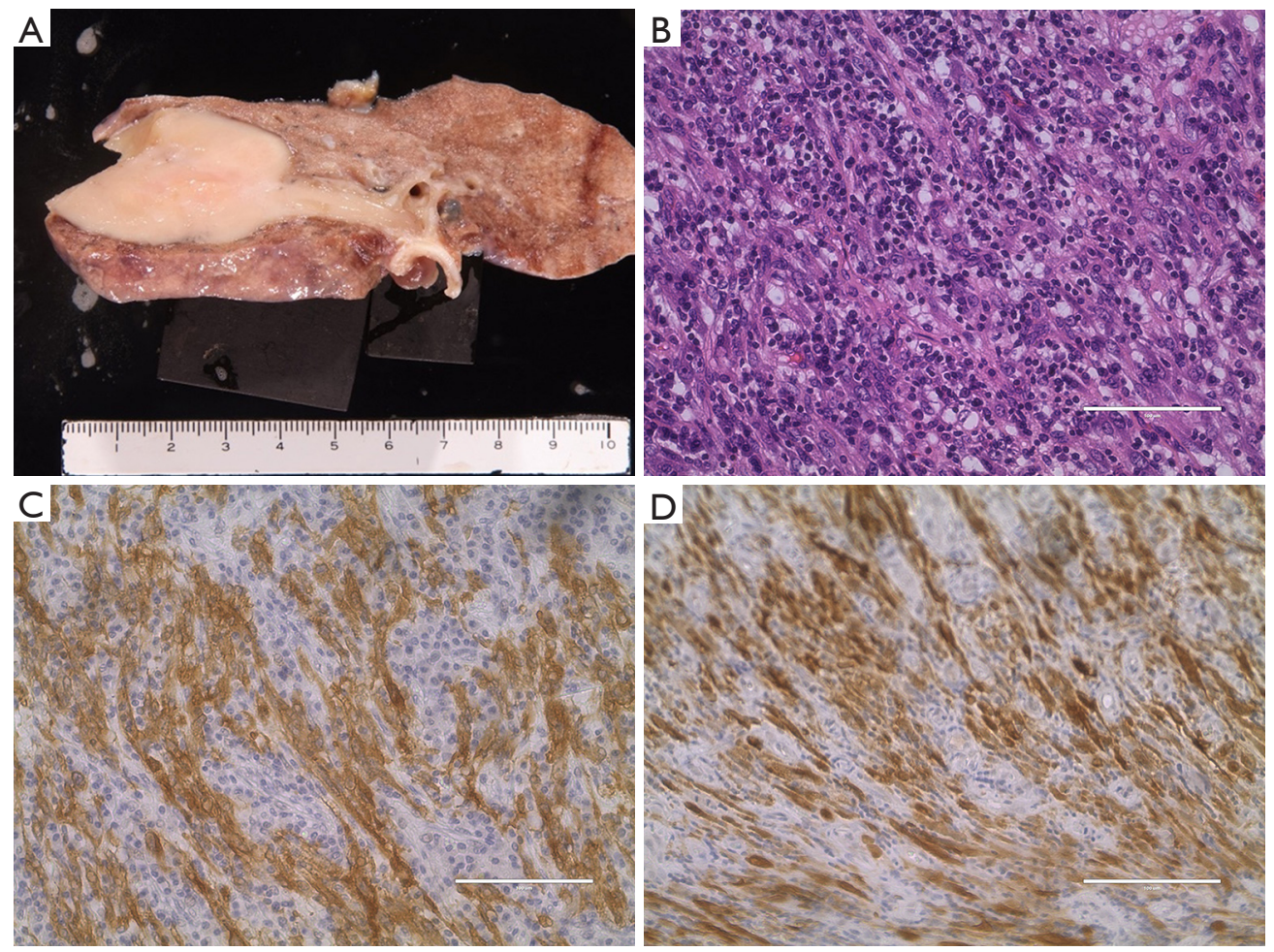

Figure 2 A macroscopic appearance with white-yellowish and boundary clearly covered with bronchial epithelium (A). Histopathological and immunohistochemistry of the permanent surgical resected tissue by hematoxylin and eosin stain ( $\times 400)(\mathrm{B})$ and ALK using the iAEP method ( $\times 400)(C)$. Histopathological and immunohistochemistry of the bronchial biopsy tissue by ALK using the iAEP method ( $\times 400)(\mathrm{D})$. iAEP, intercalated antibody-enhanced polymer.

that can occur at any age, but many are found in children, adolescents and young adults (3). ALK translocation analyses by immunohistochemistry, fluorescence in situ hybridization (FISH) or RT-PCR have become almost routine for lung adenocarcinoma patients with EGFR wild type status (1). On the other hand, pulmonary IMT is rare, but there are several reports about the ALK-rearranged tumors originating from several organs (4), and several partners of 3'-ALK have been reported (5). Recently, Lovly et al. (5) reported that gene fusions involving some kinases except for ALK, have been implicated in the pathogenesis of IMT, including ROS1 and PDGFRB. Furthermore, Antonescu et al. (6) reported that most pulmonary IMTs (83\%) were positive for fusions, either ALK or ROS1, and revealed the novel RET gene rearrangement in a patient with pulmonary IMT.

In the present case, we suspected pulmonary IMT based on findings from a pathological examination of a biopsy specimen on bronchoscopy, but we did not evaluate the ALK immune stain adequately. The IMT morphology, comprising myofibroblasts accompanied by an inflammatory infiltrate of plasma cells, variable lymphocytes, and eosinophils (7), may be confused with both reactive processes and/or malignant neoplasms on biopsy specimens. The diagnosis of pulmonary IMT by transbronchial biopsy or transthoracic needle biopsy often leads to confusion between it and fibrohistiocytic neoplasms, nodular sclerosing Hodgkin's lymphoma, NSCLC, or mediastinal fibrosis (8). The management of these diseases are different, so the correct diagnosis by biopsy is quite important for following appropriate treatment. The most cases of pulmonary IMT in adults are reported locally invasive (8), so the patients can be cured by surgical complete resection. The morphologic and adequate immunohistochemical analysis of ALK using iAEP method may be useful for correct diagnosis of pulmonary IMT using biopsy specimens. We were finally able to achieve the correct diagnosis as pulmonary IMT using the surgically resected tissue. By an adequate ALK immune stain, we confirmed ALK positivity for both the surgical resected 


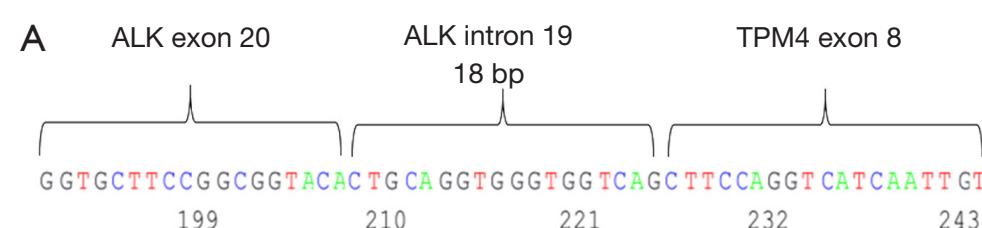

$\begin{array}{llll}199 & 210 & 221 & 232\end{array}$

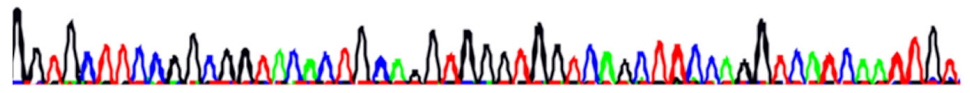

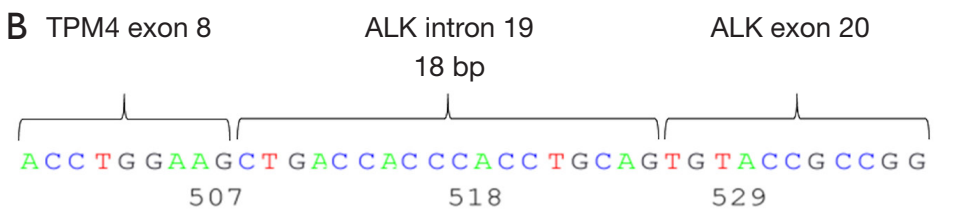

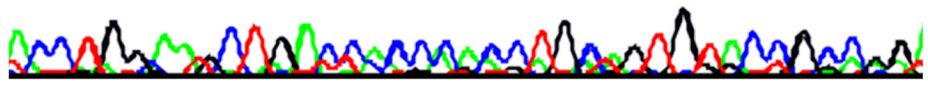

Figure 3 We extracted the RNA from the surgically resected frozen tumor tissue, and identified the TPM4-ALK as fusion gene by 5' RACE (A) and RT-PCR using a TPM4 exon primer (B). Sequencing of the TPM4-ALK by 5' RACE indicated an 18-bp inframe of the ALK intron 19 between ALK exon 20 and TPM4 exon 8. TPM4, tropomyosin alpha-4 chain; 5' RACE, 5' rapid amplification of cDNA end.

tissue and biopsy specimen. If IMT is suspected by biopsy in several organs not limited to the lung, we should evaluate the ALK expression using a sensitive ALK immune stain, like the iAEP method. In this way, we can obtain more accurate diagnosis before surgical resection and use an effective ALK inhibitor for advanced or recurrent cases.

Partners of the ALK 3 ' region have been reported for several organ tumors, and it is possible that specific fusion partners influence both the histologic features and prognosis (9). FISH can detect whether or not the tumor has an ALK rearrangement by the ALK break apart method but cannot detect the fusion partner of the ALK 3' region. We were able to detect the TPM4-ALK easily in this case using 5' RACE. 5' RACE is extremely easy method of detecting the $5^{\prime}$ partner cases of gene re-arrangement. TPM4 as the ALK fusion partner has not been described previously in pulmonary tumor. Six TPM4-ALK fusion cases have been reported in abdomen, mesentery, prostate, urinary bladder and hematologic malignant disease $(10,11)$. These reported TPM4-ALK patients were relatively young at 1 to 25 years of age. Our report is the first IMT case with TPM4-ALK fusion originating from the lung.

A molecular therapeutic drug (ALK inhibitor) was found to be extremely effective as selective therapy for lung cancer and IMT with ALK translocation (1,9). Even though, the fusion-negative IMTs occurred in adults and rarely arose in the lung or soft tissue (6), we should investigate the gene rearrangement in cases of IMT originating from all sites and determine the adequate tyrosine kinase inhibitors based on the genomic profile of the tumor.

\section{Acknowledgements}

None.

\section{Footnote}

Conflicts of Interest: The authors have no conflicts of interest to declare.

Informed Consent: Written informed consent was obtained from the patient for publication of this manuscript and any accompanying images.

\section{References}

1. Solomon BJ, Mok T, Kim DW, et al. First-line crizotinib versus chemotherapy in ALK-positive lung cancer. $\mathrm{N}$ Engl J Med 2014;371:2167-77.

2. Takeuchi K, Choi YL, Togashi Y, et al. KIF5BALK, a novel fusion oncokinase identified by an immunohistochemistry-based diagnostic system for ALK- 
positive lung cancer. Clin Cancer Res 2009;15:3143-9.

3. Gleason BC, Hornick JL. Inflammatory myofibroblastic tumours: where are we now? J Clin Pathol 2008;61:428-37.

4. Travis WD, Brambilla E, Müller-Hermelink HK, et al. Pathology and genetics of tumours of the lung, pleura, thymus and heart. Lyon: IARC Press, 2004.

5. Lovly CM, Gupta A, Lipson D, et al. Inflammatory myofibroblastic tumors harbor multiple potentially actionable kinase fusions. Cancer Discov 2014;4:889-95.

6. Antonescu CR, Suurmeijer AJ, Zhang L, et al. Molecular characterization of inflammatory myofibroblastic tumors with frequent ALK and ROS1 gene fusions and rare novel RET rearrangement. Am J Surg Pathol 2015;39:957-67.

7. Coffin CM, Hornick JL, Fletcher CD. Inflammatory myofibroblastic tumor: comparison of clinicopathologic, histologic, and immunohistochemical features including ALK expression in atypical and aggressive cases. Am J Surg

Cite this article as: Okuda K, Watanabe T, Oda R, Sakane T, Kawano O, Haneda H, Moriyama S, Nakanishi R. Pulmonary inflammatory myofibroblastic tumor with TPM4-ALK translocation. J Thorac Dis 2017;9(11):E1013-E1017. doi: 10.21037/jtd.2017.10.103
Pathol 2007;31:509-20.

8. Cerfolio RJ, Allen MS, Nascimento AG, et al.

Inflammatory pseudotumors of the lung. Ann Thorac Surg 1999;67:933-6.

9. Butrynski JE, D'Adamo DR, Hornick JL, et al. Crizotinib in ALK-rearranged inflammatory myofibroblastic tumor. N Engl J Med 2010;363:1727-33.

10. Takeuchi K, Soda M, Togashi Y, et al. Pulmonary inflammatory myofibroblastic tumor expressing a novel fusion, PPFIBP1-ALK: reappraisal of anti-ALK immunohistochemistry as a tool for novel ALK fusion identification. Clin Cancer Res 2011;17:3341-8.

11. Meech SJ, McGavran L, Odom LF, et al. Unusual childhood extramedullary hematologic malignancy with natural killer cell properties that contains tropomyosin 4--anaplastic lymphoma kinase gene fusion. Blood 2001;98:1209-16. 